\title{
Magnetocaloric effect across the coupled structural and ferromagnetic transition in $\mathrm{Pr}_{0.1} \mathrm{Ce}_{0.4} \mathrm{Sr}_{0.5} \mathrm{MnO}_{3}^{\dagger}$
}

\author{
C MADHU and A SUNDARESAN* \\ Chemistry and Physics of Materials Unit, Jawaharlal Nehru Center for Advanced Scientific Research, \\ Jakkur Post, Bangalore 560064 \\ e-mail: sundaresan $a$ jncasr.ac.in
}

\begin{abstract}
We have investigated the magnetocaloric effect across a first order structural transition coupled to the ferromagnetic transition $\left(T_{C} \sim 270 \mathrm{~K}\right)$ in $\operatorname{Pr}_{0.1} \mathrm{Ce}_{0.4} \mathrm{Sr}_{0.5} \mathrm{MnO}_{3}$ using magnetization and heat capacity measurements. The adiabatic magnetic entropy change $|\Delta S|$ obtained from magnetization measurements for a magnetic field change of $2 \mathrm{~T}$ is around $1.3 \mathrm{~J} / \mathrm{kg} \mathrm{K}$. A similar value of $|\Delta S|$ was obtained from the analysis of heat capacity data.
\end{abstract}

Keywords. Magnetocaloric effect; heat capacity; manganates; ferromagnetism.

\section{Introduction}

Recently, there has been a great deal of interest in magnetic materials showing large magnetocaloric effect (MCE). MCE is change in temperature of a magnetic material in adiabatic condition as a result of the alignment of its magnetic spins on exposure to an external magnetic field, the phenomenon which forms the basis for magnetic refrigeration. A large magnetocaloric effect was reported near room temperature in $\mathrm{Gd}$ and its alloys because of their large magnetic moment and magnetic entropy. ${ }^{1-4}$ It is interesting to note a giant MCE in $\mathrm{Gd}_{5}\left(\mathrm{Ge}_{1-x} \mathrm{Si}_{x}\right)_{4}$ where the coupled first-order structural transition and ferromagnetic transition gives a large magnetic entropy. In some materials, a first-order paramagneticferromagnetic transition has strong influence on the MCE. ${ }^{5,6}$ Very recently, perovskite manganese oxides which are known for their colossal magnetoresistance property have been shown to exhibit large MCE. ${ }^{7-15}$ They exhibit not only large magnetic entropy, large heat capacities, but also their magnetic transition temperature can be tuned over a wide range of temperature. A large magnetic entropy has been observed at the charge-ordered antiferromagnetic transition for example, in $\mathrm{R}_{0.5} \mathrm{Sr}_{0.5} \mathrm{MnO}_{3}$ (where $\mathrm{R}=\mathrm{Pr}, \mathrm{Nd}$ ). In these materials, it is well known that the applied field destroys the charge-ordered state which is accompanied by structural and magnetic transitions. ${ }^{7,12}$

\footnotetext{
${ }^{\dagger}$ Dedicated to Prof. C N R Rao on his 75th birthday
}

*For correspondence
The material $\operatorname{Pr}_{0.1} \mathrm{Ce}_{0.4} \mathrm{Sr}_{0.5} \mathrm{MnO}_{3}$, has been reported to be ferromagnetic with the transition temperature $\sim 270 \mathrm{~K}$. It has an orthorhombic structure (space group Imma) at room temperature. From the neutron diffraction measurements, a small amount of secondary phase (about $5 \%$ ) with similar composition was also found to be present in the sample. The secondary phase was reported to be tetragonal with the space group $\mathrm{I} 4 / \mathrm{mcm}{ }^{16,17}$ With decrease of temperature, the Imma phase transforms to $I 4 / \mathrm{mcm}$ at about $270 \mathrm{~K}$, which was accompanied by the ferromagnetic transition. However, a complete structural phase transition was observed only at $210 \mathrm{~K}$. At $270 \mathrm{~K}$ and $250 \mathrm{~K}$, the amount of tetragonal phase was $12 \%$ and $18 \%$ respectively. Below $210 \mathrm{~K}$, the structure remains tetragonal down to $1.5 \mathrm{~K}$. The structural transition between the orthorhombic and tetragonal phase was found to be first-order. The coupled structural and ferromagnetic transitions may influence the MCE. In order to examine this idea, we have studied the MCE in the material $\operatorname{Pr}_{0 \cdot 1} \mathrm{Ce}_{0.4} \mathrm{Sr}_{0.5} \mathrm{MnO}_{3}$.

\section{Experimental}

Polycrystalline sample, $\operatorname{Pr}_{0.1} \mathrm{Ce}_{0.4} \mathrm{Sr}_{0.5} \mathrm{MnO}_{3}$, was prepared by the conventional solid state reaction method. Stoichiometric proportion of the starting materials $\mathrm{Mn}_{2} \mathrm{O}_{3}$ (99\% Aldrich), $\mathrm{SrCO}_{3}$ (99.9\% Aldrich), $\mathrm{CeO}_{2}$ (99.995\% Aldrich), $\mathrm{Pr}_{6} \mathrm{O}_{11}$ (99.9\% Alfa Aesar) were mixed thoroughly and the mixture was heated at $900^{\circ} \mathrm{C}, 1200^{\circ} \mathrm{C}, 1300^{\circ} \mathrm{C}$ and $1400^{\circ} \mathrm{C}$ for $12 \mathrm{~h}$ with intermediate grinding. The final sintering 
was done at $1475^{\circ} \mathrm{C}$. Phase purity was checked using powder X-ray diffraction (XRD) data. Magnetization measurements were performed using Vibrating Sample Magnetometer on Physical Property Measuring System (PPMS) of Quantum Design, USA. For measuring the magnetization $(\mathrm{M})$ versus temperature (T) curve, sample was warmed slowly, stabilized at the desired temperature, and $\mathrm{M}$ was measured. The heat capacity measurement was performed with PPMS using Heat Capacity Option, which is based on thermal relaxation of the sample.

\section{Results and discussion}

The XRD pattern showed that the structure is orthorhombic as reported earlier. ${ }^{16}$ The presence of secondary phase of a similar composition could not be confirmed by the XRD. However, we believe that the presence of such a small amount of tetragonal phase may not influence the results significantly. The magnetization versus temperature curves, under different applied field conditions, for $\operatorname{Pr}_{0 \cdot 1} \mathrm{Ce}_{0.4} \mathrm{Sr}_{0.5} \mathrm{MnO}_{3}$ is shown in figure 1 . The ferromagnetic transition temperature obtained from magnetization data measured at $0.005 \mathrm{~T}$ is around $270 \mathrm{~K}$ as seen in the inset of figure 1. Magnetization curves shown in figure 1 were obtained by first cooling the sample in presence of magnetic field to $160 \mathrm{~K}$ and then warming the sample to $320 \mathrm{~K}$ in step of $2 \mathrm{~K}$. At each temperature the sample was stabilized for $30 \mathrm{~s}$ before the data were collected.

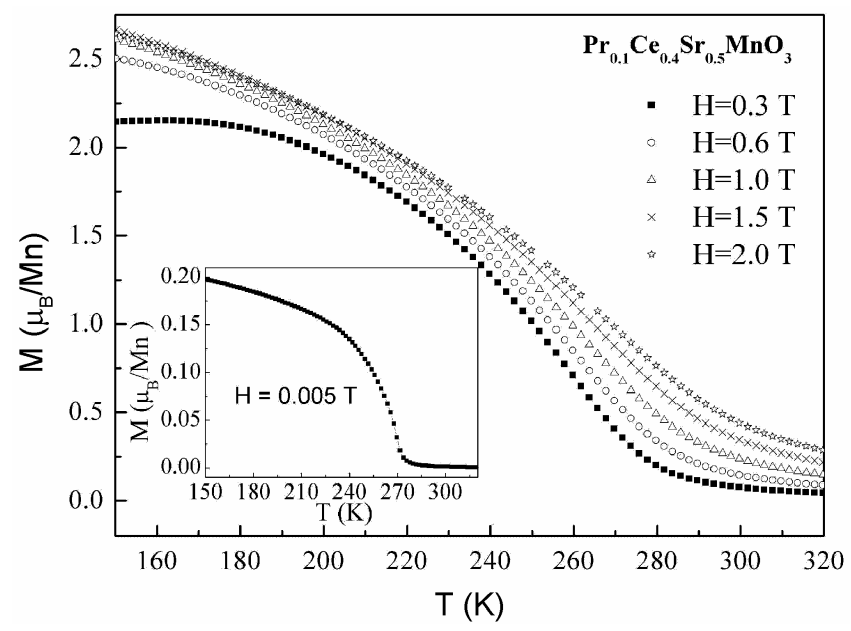

Figure 1. Magnetization versus temperature curves, in presence of applied field of $0.3 \mathrm{~T}, 0.6 \mathrm{~T}, 1 \mathrm{~T}, 1.5 \mathrm{~T}$ and $2 \mathrm{~T}$. Inset displays the sharp magnetic transition observed in presence of $0.005 \mathrm{~T}$.
Figure 2 shows the magnetization as a function of magnetic field at different temperatures around $T_{C}$. In inset we have plotted the Arrott plot that confirms the magnetic transition to be from paramagnetic $(\varepsilon<0)$ to ferromagnetic $(\varepsilon>0)$ and also the $T_{C}$ obtained from this plot, where $\varepsilon=0$, is $265 \mathrm{~K} .^{18,19}$

We employ indirect method to calculate MCE as described in earlier reports (Neumeier et $a l^{8}$; Dinesen $\left.e t a l^{9}\right)$. The origin of the magnetocaloric effect is due to coupling of magnetic sub-lattice with the magnetic field, which results in magnetic entropy

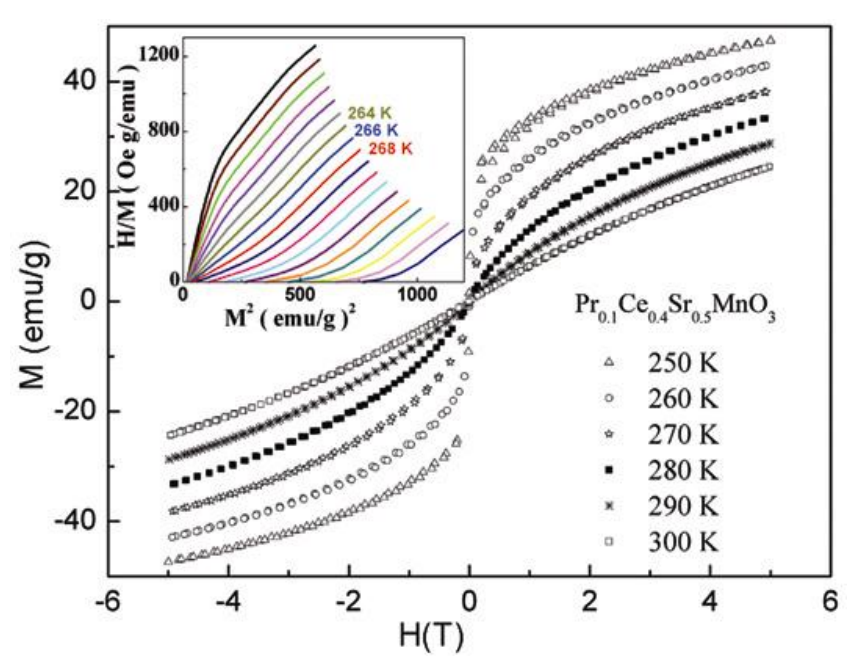

Figure 2. $M$ versus $H$ curve measured at different temperatures across the magnetic transition region. Inset is Arrott plot from $258 \mathrm{~K}$ to $276 \mathrm{~K}$ at step of $2 \mathrm{~K}$.

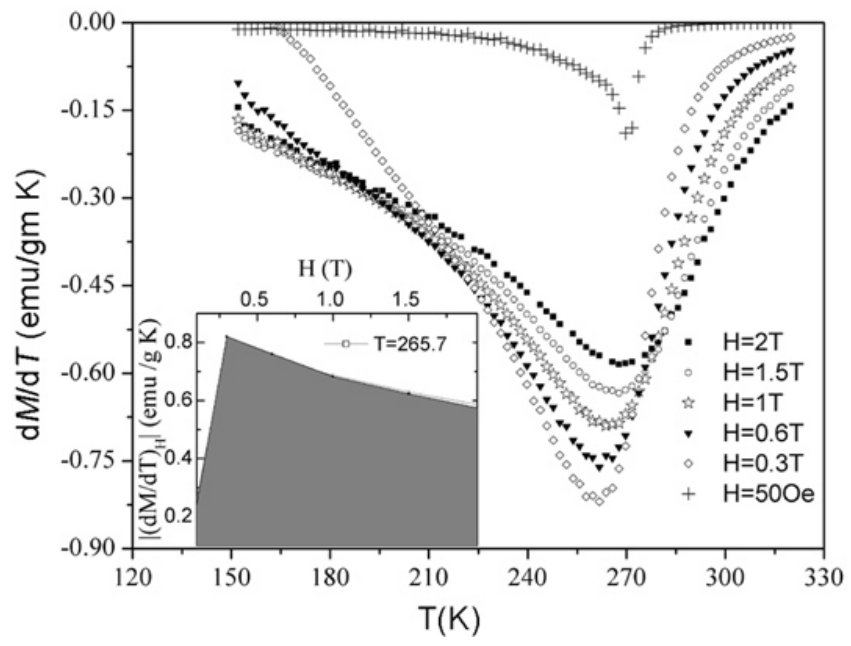

Figure 3. The derivatives of magnetization with respect to temperature, $(\partial M / \partial T)_{H}$ data at different applied fields are displayed. Inset shows $\left|(\partial M / \partial T)_{H}\right|$ versus $H$ plot at $265.7 \mathrm{~K}$. The shaded area under the curve corresponds to the $|\Delta S|$ value. 
change $(\Delta S)$ which in turn gives rise to change in temperature $\left(\Delta T_{\mathrm{ad}}\right)$ under adiabatic condition. These two thermodynamic quantities depend on temperature and are measured on the magnetic field. Thus, the magnetocaloric effect can be evaluated by measuring the magnetization and heat capacity with the use of the theory of thermodynamics. The Maxwell relation (1) relates the entropy change and magnetization,

$$
\left(\frac{\partial M}{\partial T}\right)_{H}=\left(\frac{\partial S}{\partial H}\right)_{T}
$$

where $M$ is the magnetization, $T$ is the temperature, $S$ is the magnetic entropy and $H$ is the magnetic field. On integrating the above equation over the field range we get,

$$
\Delta S=\int_{0}^{H_{\max }}\left(\frac{\partial M}{\partial T}\right)_{H} \mathrm{~d} H,
$$

which can be used to obtain the change in the magnetic entropy. The total entropy $|\Delta S|$, is determined by taking the derivative of magnetization data and find the area under the $(\partial M / \partial T)_{H}$ versus $H$ curve. From (2), it is evident that this approximates the area under the $(\partial M / \partial T)_{H}$ versus $H$ curve as rectangle. To find the maximum in $|\Delta S|$ more precisely, derivatives at number of magnetic fields, $0.005 \mathrm{~T}, 0.3 \mathrm{~T}$, $0.6 \mathrm{~T}, 1 \mathrm{~T}, 1.5 \mathrm{~T}$ and $2 \mathrm{~T}$ were determined as shown in figure 3 . The obtained values of $|\Delta S|$ are plotted as a function of temperature as shown in figure 4 for an applied field of $2 \mathrm{~T}$. It is obvious from this figure

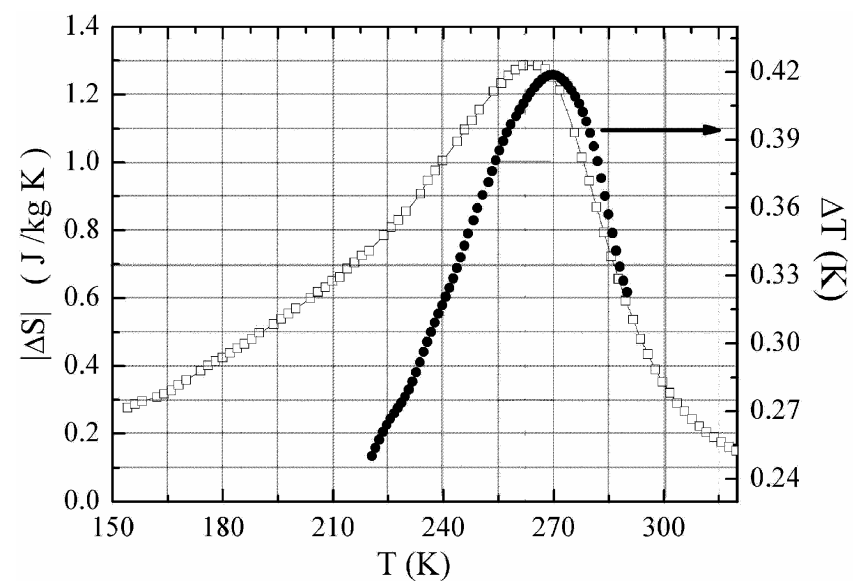

Figure 4. The value of $|\Delta S|$ versus temperature calculated from magnetization data (in open circle). The change in temperature $\Delta T$ versus $T$ calculated from heat capacity data using equation 4 (closed circle). that there is peak in $|\Delta S|$ which is defined as $|\Delta S|_{\max }$. It is interesting to note that the $|\Delta S|_{\max }$ occurs near the ferromagnetic transition temperature $T_{C}$.

Increasing magnetic field shifts $T_{C}$ to higher temperature but broadens the $M(T)$ curve due to fluctuation effects. ${ }^{8}$ Further, it shifts the maximum in $(\partial M / \partial T)_{H}$ to higher temperature and reduce the value of $|\Delta S|_{\max }$. The value of $|\Delta S|_{\max }$ obtained for a magnetic field change of $2 \mathrm{~T}$ is about $1.3 \mathrm{~J} / \mathrm{kg} \mathrm{K}$ which is moderate. In contrast to the large magnetic entropy observed in many materials where there is a firstorder structural transition near the magnetic transition, the present system shows relatively lower value of magnetic entropy. This may be due to lack of complete structural phase transition near the ferromagnetic transition as discussed in the introduction.

Although measurements of $|\Delta S|_{\max }$ from magnetization data provide a good guide to the magnitude of magnetocaloric effect, it is essential that heat capacity measurements be used as well to make a complete evolution of MCE. Heat capacity data collected at zero and $2 \mathrm{~T}$ field in the temperature range 220 $300 \mathrm{~K}$ are plotted in the inset of figure 5. These data were used to determine $|\Delta S|$ and compare its value to the one obtained from magnetization data.

The magnetocaloric effect is the change in temperature $\Delta T=[T(H)-T(0)]$ of magnetic material in the adiabatic condition with the application of the external magnetic field. ${ }^{13}$ This effect originates due to the change of magnetic entropy of magnetic material in the presence of magnetic field. For ferromag-

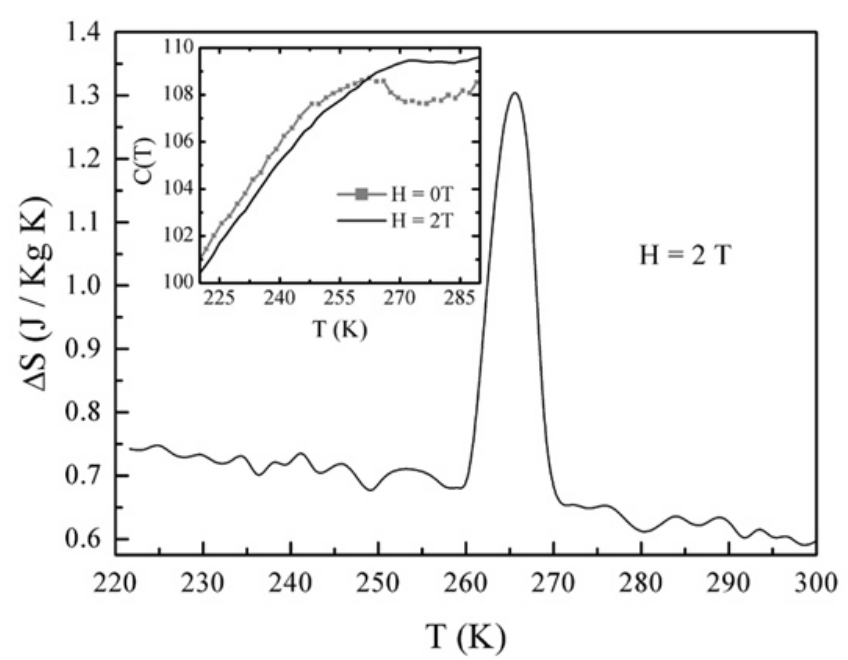

Figure 5. The magnetocaloric effect obtained from the heat capacity data. One can observe that the $|\Delta S|$ value near transition temperature is around $1.3 \mathrm{~J} / \mathrm{kg} / \mathrm{K}$. The heat capacity for zero and $2 \mathrm{~T}$ field is shown in the inset. 
netic materials close to $T_{C}$ magnetic entropy decreases with increasing magnetic field and in an adiabatic condition that gives rise to an increase in temperature, positive $\Delta T_{\mathrm{ad}}$. The magnitude of $\Delta T_{\mathrm{ad}}$ is maximum, where the rate of change of magnetization (as a function of temperature) is high. In order to calculate MCE from heat capacity data, the $\mathrm{C} / \mathrm{T}$ versus $\mathrm{T}$ data in the temperature range $220-300 \mathrm{~K}$, in presence of constant magnetic field, are integrated to find the temperature dependent entropy change $\Delta S_{T}$ using,

$$
\Delta S_{T}(H)=\int_{T_{\min }}^{T_{\max }}\left(\frac{C(T, H)}{T}\right) \mathrm{d} T .
$$

$|\Delta S|$ is then determined from $\left|\Delta S_{\mathrm{T}, \mathrm{H}=2 \mathrm{~T}}-\Delta S_{\mathrm{T}, \mathrm{H}=0 \mathrm{~T}}\right|$; the resulting data is plotted in figure 5 , illustrating good agreement between the two methods. Further, we calculate the temperature change $\left|\Delta T_{\text {ad }}\right|$ realized by a change in magnetic field by using,

$$
\Delta T_{\mathrm{ad}}=-\int_{0}^{H_{\max }}\left(\frac{T}{C(T, H)}\right)\left(\frac{\partial M}{\partial T}\right)_{H} \mathrm{~d} H .
$$

$C(T, H)$ in the above equation refers to the total heat capacity. A plot of $|\Delta T|$ versus $T$ is shown in figure 4 , and it exhibits a broad peak near the transition. The value $|\Delta T|$ was found to be $0.42 \mathrm{~K}$ at $T=270 \mathrm{~K}$. Finally, we calculate the Relative Cooling Power (RCP), ${ }^{9}$ which gives the cooling power of a refrigerator using the relations,

$$
R C P(S)=\left|\Delta S_{M}\right|_{\max } \times W\left(\Delta S_{M}\right),
$$

where, $W\left(\Delta S_{\mathrm{M}}\right)$ denotes the full width at half maxima of magnetic entropy change curve. In the present case the RCP(S) is found to be $89 \mathrm{~J} \mathrm{~kg}^{-1}$, which is comparable to those reported.

\section{Conclusion}

The magnitude of the magnetocaloric effect determined for $\operatorname{Pr}_{0.1} \mathrm{Ce}_{0.4} \mathrm{Sr}_{0.5} \mathrm{MnO}_{3}$ using magnetization and heat capacity data for a magnetic field change of $2 \mathrm{~T}$ is moderate $(\Delta S=1.3 \mathrm{~J} / \mathrm{K} \mathrm{kg})$ compared to some of the manganese perovskite. However, the values of entropy change obtained from both methods are in good agreement. The relatively lower value of magnitude of the magnetocaloric effect may be due to incomplete structural transition near $T_{C}$.

\section{References}

1. Pecharsky V K and K A Gschneidner Jr 1997 Phys. Rev. Lett. 784494

2. Rawat R and Das I 2006 J. Phys.: Condens. Matter 181051

3. Giguere A, Foldeaki M, Ravi Gopal B, Chahine R, Bose T K, Frydman A and Barclay J A 1999 Phys. Rev. Lett. 832262

4. Pecharsky V K, Tang H, Pecharsky A O, Schlagel D L, Lograsso T A and Gschneidner Jr K A $2003 J$. Appl. Phys. 938298

5. von Ranke P J, de Campos A, Caron A, Coelho A A, Gama S and de oliveira N A 2004a Phys. Rev. B70 94410

6. von Ranke P J, de Oliveira N A and Gama S 2004b Phys. Lett. A320 302

7. Chen P, Du Y W and Ni G 2000 Europhys. Lett. 52 589

8. Neumeier J J, Hirotoshi Terashita and Garbe J J 2004 Phys. Rev. B70 94403

9. Dinesen A R, Linderoth S and Morup S $2005 \mathrm{~J}$. Phys.: Condens. Matter. 176257

10. Hong Zhu, Hao Song and YuHeng Zhang $2002 \mathrm{Appl}$. Phys. Lett. 813416

11. Wolff Fabris F, Pekala M, Drozd V, Ru-Shi Liu, Ausloos M, J-F. Fagnard and Ph. Vanderbemden $2007 \mathrm{~J}$. Appl. Phys. 101103904

12. Sande P, Hueso L E, Miguens D R, Rivas J, Rivadulla F and Lopez Quintela M A 2001 Appl. Phys. Lett. 792040

13. Pecharsky V K, Gschneidner Jr K A and Tsokol A O 2005 Rep. Prog. Phys. 681479

14. Sheng-Bo Tian, Manh-Huong Phan, Seong-Cho Yu and Nam Hwi Hur 2003 Physica B327 221

15. de Oliveira N A 2007 Appl. Phys. Lett. 90052501

16. Sundaresan A, Caignaert V, Maignan A, Suard E and Raveau B 1999 Phys. Rev. B60 533

17. Sundaresan A, Caignaert V, Raveau B and Suard E 1994 Solid State Comm. 104489

18. Anthony Arrott 1957 Phys. Rev. 1081394

19. Anthony Arrott and John E. Noakes 1967 Phys. Rev. Lett. 19786 